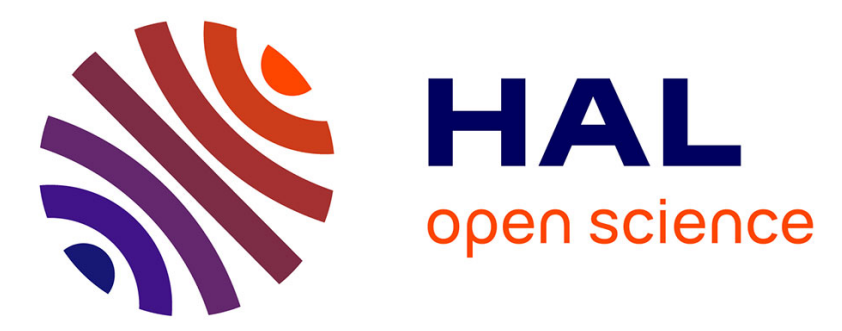

\title{
Comparison of four visual analytics techniques for the visualization of adverse drug event rates in clinical trials Jean-Baptiste Lamy
}

\section{To cite this version:}

Jean-Baptiste Lamy. Comparison of four visual analytics techniques for the visualization of adverse drug event rates in clinical trials. International Conference Information Visualisation, Sep 2020,

Vienna, Austria. hal-02968289

\section{HAL Id: hal-02968289 \\ https://hal.science/hal-02968289}

Submitted on 15 Oct 2020

HAL is a multi-disciplinary open access archive for the deposit and dissemination of scientific research documents, whether they are published or not. The documents may come from teaching and research institutions in France or abroad, or from public or private research centers.
L'archive ouverte pluridisciplinaire HAL, est destinée au dépôt et à la diffusion de documents scientifiques de niveau recherche, publiés ou non, émanant des établissements d'enseignement et de recherche français ou étrangers, des laboratoires publics ou privés. 


\title{
Comparison of four visual analytics techniques for the visualization of adverse drug event rates in clinical trials
}

\author{
Jean-Baptiste Lamy \\ Université Sorbonne Paris Nord, LIMICS, INSERM, UMR 1142, F-93000, Bobigny, France \\ Laboratoire de Recherche en Informatique, CNRS/Université Paris-Sud/Université Paris-Saclay, Orsay, France \\ Email: jean-baptiste.lamy@univ-paris13.fr
}
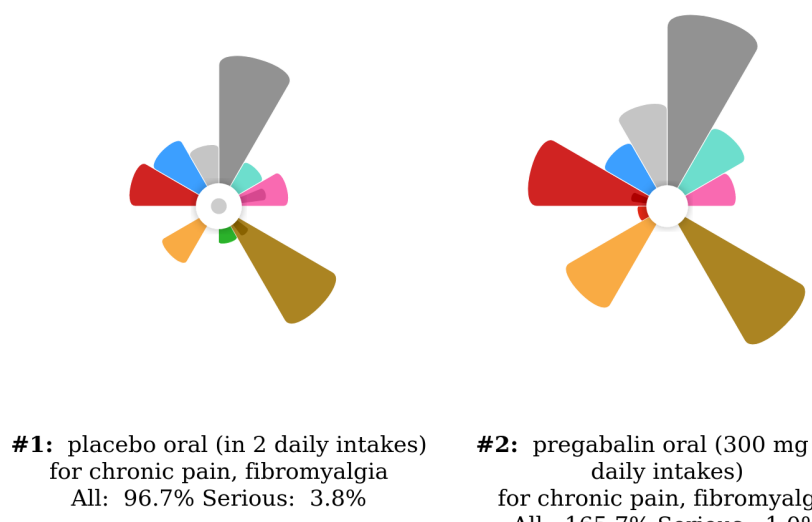
\#2: pregabalin oral (300 mg in 2 daily intakes)
for chronic pain, fibromyalgia
All: $165.7 \%$ Serious: $1.0 \%$

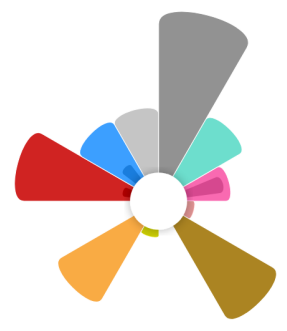
\#3: pregabalin oral (450 $\mathrm{mg}$ in 2 daily intakes)
for chronic pain, fibromyalgia
All: $163.7 \%$ Serious: $4.4 \%$

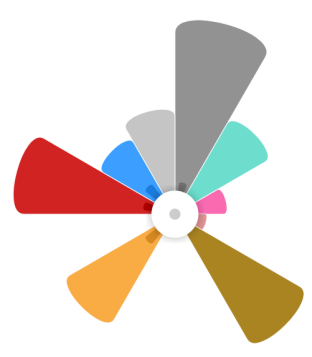
\#4: pregabalin oral (600 mg in 2 daily intakes)
for chronic pain, fibromyalgia All: $180.1 \%$ Serious: $2.6 \%$

Figure 1. Visualization of the ADE rates of four groups in a clinical trial, using area-proportional flower glyphs.

\begin{abstract}
Drugs are evaluated and compared during clinical trials, and the observed adverse drug events are recorded. Many trial results are available online in trial registries, such as ClinicalTrials.gov. However, this data remains little used currently, due to the volume and the tabular presentation.

In this paper, we focus on the visualization of adverse drug event rates, aggregated in 13 anatomical categories and 2 levels of seriousness. We designed four interactive visual analytics: horizontal stacked bar chart with labels, vertical stacked bar chart, area-proportional flower glyphs and star glyphs. We compare the four approaches, analytically and through a preliminary user study aimed at determining the user preferred visual analytics, and we identify their advantages and disadvantages. We conclude that the best options are the horizontal stacked bar chart, which allows labels to help identify the categories of adverse drug events, and the area-proportional flower glyph, which permits a better visualization of small values when very high values are present.
\end{abstract}

Index Terms-Visual analytics, clinical trial, drug adverse events, flower glyph, star glyph.

\section{INTRODUCTION}

Clinical trials are experimental studies aimed at evaluating a medical procedure or product. In particular, drugs are evaluated during clinical trials. Each trial includes one or several groups of similar patients, and each group receives a different drug treatment (which may be a placebo). For each group, the outcomes are measured, in terms of efficacy and safety. More specifically, regarding safety, all adverse events observed are recorded, since they might have been caused by the drug treatment received.

Today, more than 42,000 trial results are available online at ClinicalTrials.gov. This data includes outcomes, but also the detailed tables of Adverse Drug Events (ADE) for each group. These tables contain important and interesting clinical evidence for comparing drugs, however, they are far too voluminous for being used as such.

Visual analytics [1] have been proved to be efficient for presenting voluminous data in a synthetic and visual way, in order to facilitate the discovery of new insight from the data. Among many other domains, visual analytics have been applied to drug clinical properties. In the literature, stacked bar charts were proposed for summing visually the ADE rates of the various drugs in a drug orders [2]. In previous study, we proposed visual approaches for the comparison of drug properties such as contraindications and adverse effects [3], [4] and for the comparison of properties of antibiotics found in a knowledge base [5], [6].

In this paper, we describe the design of four visual analytics techniques for the visualization of ADE rates observed in clinical trials: horizontal stacked bar chart, vertical stacked bar chart, flower glyph and star glyph. We compare the four approaches, analytically and through a preliminary user study 


\begin{tabular}{|l|l}
\hline & Psychology and psychiatry \\
\hline & Nervous system \\
& Sensory system \\
& Musculo-skeletic system \\
& Skin and subcutaneous tissue \\
& Digestive system \\
Genital system and reproduction \\
Urinary system \\
\hline & Endocrine system, metabolism and nutrition \\
\hline & $\begin{array}{l}\text { Blood and immune system } \\
\text { Cardiovascular system }\end{array}$ \\
\hline & $\begin{array}{l}\text { Respiratory system } \\
\text { Unclassified }\end{array}$ \\
\hline
\end{tabular}

Figure 2. The $13 \mathrm{ADE}$ categories and their associated colors.

aimed at determining the user preferred visual analytics, and identify their advantages and disadvantages.

The rest of the paper is organized as follows. Section II describes related works on visual analytics and glyphs. Section III presents the design of the four visual analytics techniques for ADE rates. Section IV shows the resulting visual analytics and gives examples. Section V presents the preliminary user study. Finally, section VI discusses the results of the presented works and their limits, and concludes with perspectives.

\section{RELATED WORKS}

Many visual analytics techniques exist, including wellknown diagrams such as stacked bar charts or pie charts. A more sophisticated technique is glyph [7], [8]: they represent each piece of data by a small icon. The glyph includes several parts, in terms of color, length, orientation, etc.

Two particular types of glyphs have been proposed for representing tuples of numerical values: the star glyph and the flower glyph. In the star glyph (or stardinates) [9], [10], three or more axes are considered. All axes share the same origin, but each axis is oriented in a different direction. The glyph represents a tuple of numerical values (one per axis), and is a closed polygon, obtained by joining the points corresponding to the position of each value on its axis. Usually, each glyph has its own set of axes, but several star glyphs can also be superposed.

Flower glyphs (also known as Nightingale's Rose plot) [11], [12], [13] are flower-shaped. The glyph has several petals. It represents a tuple of numerical values (one per petal), and the length of each petal is proportional to the corresponding value. Petals can be colored. G. Pilato et al. [14] proposed the addition of a central region to the flower glyph. The ring glyph [15] is quite similar to the flower glyph, but petals are replaced by round bubbles. These bubbles may overlap each other. The leaf glyph [16] is another botanic-inspired glyph.

\section{VISUAL ANALYTICS DESIGN}

ADE were classified in 13 anatomico-functional categories: cardiovascular, neurologic,... plus an "unclassified" category for ADE that are not specified in terms of anatomy (e.g. general symptoms like asthenia or cancer without precision of the organ involved). Each ADE was classified in one or two categories, using the MedDRA (Medical Dictionary for Drug Regulatory Activities) standard medical terminologies that is recommended for the description of ADE in ClinicalTrials.gov. In addition to the category, two levels of seriousness are present in ClinicalTrials.gov: serious ADE vs non-serious ADE. Thus, for each group in a clinical trial, we need to visualize $13 \times 2=26$ real values.

We associated a specific color with each category (Figure 2 ), and we tried to use colors often associated with the corresponding organs or systems, e.g. yellow for the urinary system, red for the cardiovascular system, gray for the nervous system, or brown for the digestive system. Arbitrary colors were used when no such color exist, e.g. green for the reproductive system. The unclassified category was associated with the white and/or the dark gray color. When relevant for the visual techniques, we used a darker color for serious ADE.

We designed horizontal and vertical stacked bar charts with 13 bars (one per category), with two stacks: one for serious ADE (at the bottom/left for vertical/horizontal bar charts, with a darker color) and one for non-serious ADE (at the top/right). We ordered categories so as to position medically related categories next to each other, e.g. the respiratory system was placed next to the cardiovascular system. Unclassified ADE were added as a last black bar, on the right/bottom. In addition, in the horizontal stacked bar chart, we added textual short labels indicating the ADE category, e.g. "cardiovascular".

We designed the flower glyph as having 12 petals corresponding to the $12 \mathrm{ADE}$ anatomic categories, and a central white region representing unclassified ADE. Serious ADE were represented by a darker region inside the 12 petals and the central region. For deciding the position of each category on the flower glyph, we considered the position of the corresponding organs in the human body, e.g. the brain is the largest nervous organ is located at the top of the body, thus the "nervous" category was placed at the top. On the contrary, the urinary system is at the bottom. We also tried to position related categories next to each other, as above.

Contrary to what was found in the literature, we used areaproportional flower glyph: the area of the petal is proportional to the ADE rate, and not the length of the petal. Similarly, the central region and the darker regions for serious ADE are areaproportional to the ADE rate. The use of area-proportionality was motivated by the fact that the human eye is known to be more sensitive to area rather than to distance [17], but also by the fact that ADE rates are often non-linear, with many small values and a few high values. Consequently, areaproportionality allows showing more details on small values. 
We designed the star glyph by considering 13 axes, one per ADE category. Each axis was colored using the category color, and we added a colored circle at the axis end to improve the color visibility. Categories were presented in the same order as in the flower glyph, with the addition of the unclassified category (since star glyph has no central region). We superposed two polygons on the star glyph: a gray polygon for serious+non-serious $\mathrm{ADE}$ rate and a red polygon for serious ADE rate only. Contrary to the previously described flower glyph, the star glyph is linear, i.e. the position of the polygon's vertices on the axes is proportional to the ADE rate.

To permit a better comparison, we devoted to each visual analytics a screen surface that is roughly equivalent.

\section{RESUlts}

We used as an example the trial entitled "Randomized, Double-Blind, Placebo-Controlled Trial Of Pregabalin In Patients With Fibromyalgia" (found in ClinicalTrials.gov, NCTID: NCT00333866, available at https://clinicaltrials.gov/ct2/show/study/NCT00333866). This trial tests the use of pregabalin, an anti-epileptic drug frequently prescribed for pain, for the treatment of pain caused by fibromyalgia. Three doses of pregabalin are tested in the trial $(300 \mathrm{mg}, 450 \mathrm{mg}, 600 \mathrm{mg}$ ), as well as a placebo. There are thus four groups of patients to compare.

Figure 3, 4, 5 and 1 show the ADE rates for the four groups of this trial, with vertical stacked bar chart, horizontal stacked bar chart, star glyph and flower glyph, respectively. We can see that the readability of the star glyph is limited. First, serious ADE are almost invisible, due to their low rate. Second, high rates surrounded by zero values are almost invisible (e.g. the rate of digestive adverse events is high but difficult to see, because the two contiguous categories, skin and reproduction, have very low rates).

We added interactivity to the proposed visual analytics, in two forms. First, we used the common "details-on-demand" method : additional details are displayed when the mouse cursor is over a bar, a petal or an axis (depending on the visual analytics). A pop-up label displays the name of the ADE category selected, the rate of ADE in this category, the most frequent $\mathrm{ADE}$ observed, the rate of serious $\mathrm{ADE}$ in the category and the most observed serious ADE. Figure 6 shows an example of details-on-demand.

Second, we added an option for selecting a patient group in order to compare to the other. When activated, this option draws the outline of the selected bar chart, flower glyph or star glyph on top of the others. This outline is expected to facilitate the fine comparison between the patient groups and to help finding small difference. Figure 6 shows an example of this option with flower glyphs.

\section{USER OPINION}

The four proposed visual analytics were presented to 8 persons in the field of medical informatics and/or computer science. The study was performed online, using the Firefox or Chrome web browser. The objective of the study was briefly introduced, then each visualization technique was presented, using Figure 4, 3, 1 and 5, in that order. A color key was also provided. Finally, each participant was asked to rank the four visual analytics, and to provide personal comments.

For each visualization technique, we computed a score, obtained by giving 3 points when the technique was ranked in the first position by a participant, 2 points when it was ranked in the second position, 1 point when it was ranked third, and 0 otherwise. We then computed the average scores. The resulting scores are 2.0 for the flower glyph, 1.375 for the horizontal stacked bar chart, 1.25 for the vertical one and 1.25 for the star glyph.

The analysis of participant comments reveals interesting information. Persons preferring the star glyph justified their choice by the fact that the surface of the star glyph gives an immediate idea of the ADE rate, which is actually not the case: star glyphs are not area- proportional. Thus, the star glyph is misleading and may lead to wrong interpretation. Persons preferring the horizontal bar chart appreciated the textual labels that helped to identify the 13 ADE categories. Persons preferring the flower glyphs mentioned their visual attractiveness but also their ability to favor small values and their area-proportionality. Finally, few arguments were given in favor of the vertical bar chart, excepted the fact that they are more commonly used than the horizontal one.

Table I summarizes the advantages and disadvantages of the four visualization techniques. In particular, the readability of star glyph is particularly poor in this application.

\section{DISCUSSION}

In the literature, few works focused on the visualization of trial results data, despite the fact that more and more such data is available online on registries such as ClinicalTrials.gov. A graph visualization of chemotherapy treatment regimens was proposed by J. Warner et al. [18]. Maps, diagrams and parallel coordinates were proposed by J. Sjöbergh et al. for the visualization of the data of a given patient participating in a trial [19].

In the literature, flower glyphs are usually lengthproportional. However, in this work, we have seen that areaproportional flower glyph can be particularly interesting for enhancing the difference between small values. Figure 8 shows the same data as in Figure 1, but with flower glyph with petal length being proportional to ADE rate (instead of petal surface), thus being linear. Compared to Figure 1, we can see that small values are much more difficult to identify. In particular, in the glyph on the left for placebo, two petals are huge (nervous and digestive systems, in gray and brown respectively), while all other are very small. These small petals may be difficult to read, but also to select for obtaining more information with the detail-on-demand interactivity. 


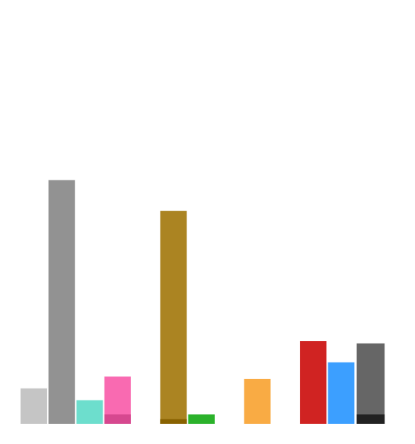

\# 1: placebo oral (in 2 daily intakes) for chronic pain, fibromyalgia All: $96.7 \%$ Serious: $3.8 \%$

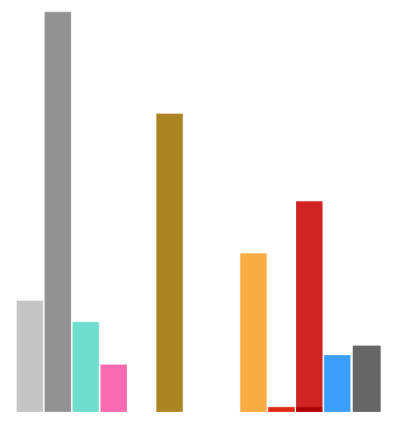

\#2: pregabalin oral (300 mg in 2 daily intakes)

for chronic pain, fibromyalgia All: $165.7 \%$ Serious: $1.0 \%$

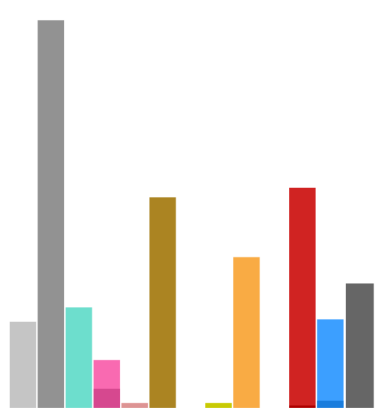

\#3: pregabalin oral ( $450 \mathrm{mg}$ in 2 daily intakes)

for chronic pain, fibromyalgia All: $163.7 \%$ Serious: $4.4 \%$

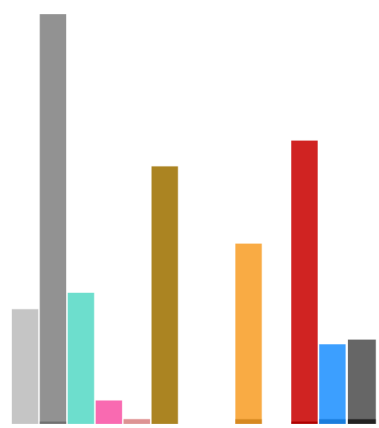

\#4: pregabalin oral (600 $\mathrm{mg}$ in 2 daily intakes)

for chronic pain, fibromyalgia

All: $180.1 \%$ Serious: $2.6 \%$

Figure 3. Visualization of the ADE rates of four groups in a clinical trial, using vertical stacked bar charts.

psychology/psychiatry
nervous
sensory
musculo-skeletic
skin/subcutaneous tissue
digestive
genital/reproduction
urinary
endocrine/metabolism/nutrition
blood/immune
$\quad$ vascular
respiratory

\#1: placebo oral (in 2 daily intakes) for chronic pain, fibromyalgia All: $96.7 \%$ Serious: $3.8 \%$

\begin{tabular}{l} 
psychology/psychiatry \\
nervous \\
sensory \\
musculo-skeletic \\
skin/subcutaneous tissue \\
digestive \\
genital/reproduction \\
urinary \\
endocrine/metabolism/nutrition \\
blood/immune \\
\hline respiratory
\end{tabular}

\#2: pregabalin oral (300 mg in 2 daily intakes)

for chronic pain, fibromyalgia

\begin{tabular}{l} 
psychology/psychiatry \\
nervous \\
\hline sensory \\
musculo-skeletic \\
skin/subcutaneous tissue \\
digestive \\
genital/reproduction \\
|urinary \\
endocrine/metabolism/nutrition \\
blood/immune \\
\hline \\
\hline respiratory
\end{tabular}

\#3: pregabalin oral (450 mg in 2
daily intakes) for chronic pain, fibromyalgia

\begin{tabular}{l} 
psychology/psychiatry \\
nervous \\
\hline sensory \\
musculo-skeletic \\
skin/subcutaneous tissue \\
digestive \\
genital/reproduction \\
urinary \\
endocrine/metabolism/nutrition \\
blood/immune \\
\hline respiratory
\end{tabular}

\#4: pregabalin oral (600 mg in 2 daily intakes)

for chronic pain, fibromyalgia

All: $180.1 \%$ Serious: $2.6 \%$

Figure 4. Visualization of the ADE rates of four groups in a clinical trial, using horizontal stacked bar charts with labels.

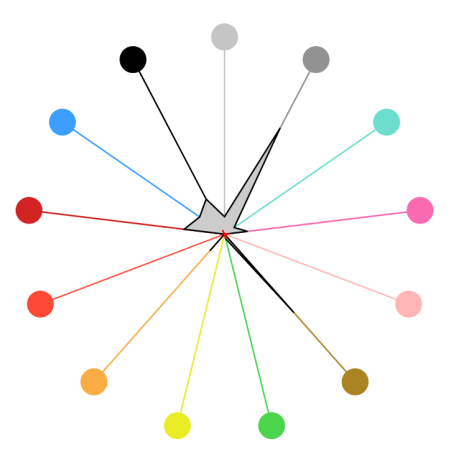

\#1: placebo oral (in 2 daily intakes) for chronic pain, fibromyalgia All: $96.7 \%$ Serious: $3.8 \%$

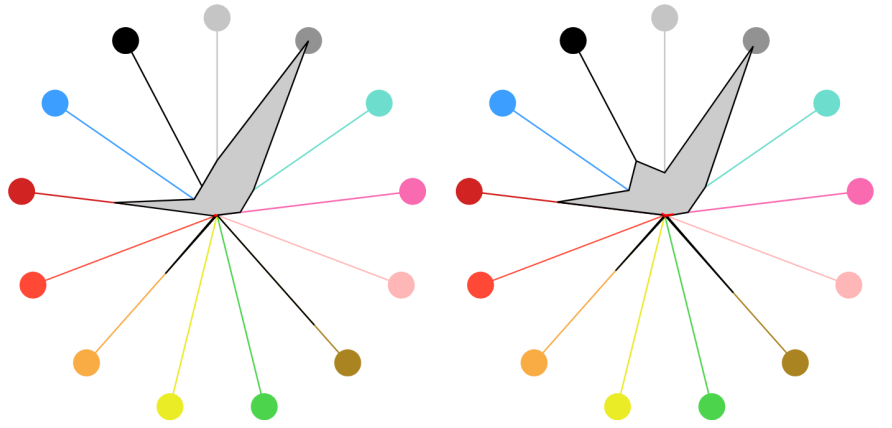

\#2: pregabalin oral (300 $\mathrm{mg}$ in 2 daily intakes)

for chronic pain, fibromyalgia All: $165.7 \%$ Serious: $1.0 \%$
\#3: pregabalin oral (450 mg in 2 daily intakes)

for chronic pain, fibromyalgia

All: $163.7 \%$ Serious: $4.4 \%$

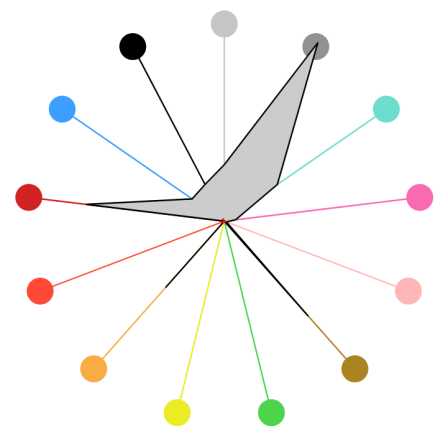

\#4: pregabalin oral (600 mg in 2 daily intakes)

for chronic pain, fibromyalgia

All: $180.1 \%$ Serious: $2.6 \%$

Figure 5. Visualization of the ADE rates of four groups in a clinical trial, using star glyphs. 


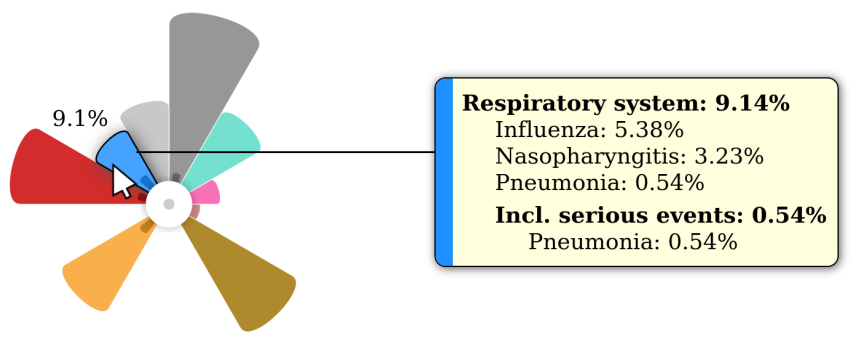

$\Delta$

\#4: pregabalin oral (600 $\mathrm{mg}$ in 2 daily intakes)

for chronic pain, fibromyalgia

All: $180.1 \%$ Serious: $2.6 \%$

Figure 6. Flower glyph showing details on demand when the mouse cursor is over the blue petal.
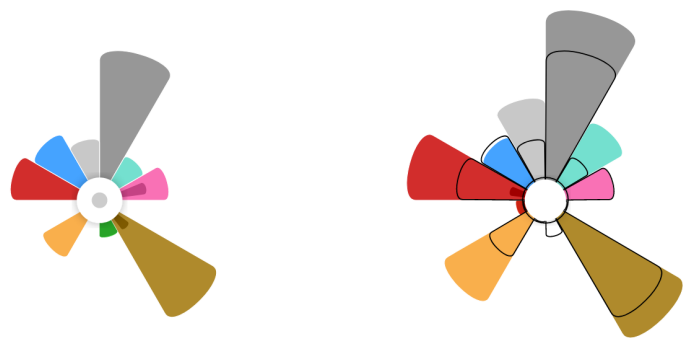

\#2: pregabalin oral ( $300 \mathrm{mg}$ in 2 daily intakes) for chronic pain, fibromyalgia All: $165.7 \%$ Serious: $1.0 \%$
Figure 7. Flower glyph with the comparison option activated, showing the outline of the first glyph on top of the second.

The visual attractiveness of flower glyphs, compared to bar charts, might be explained as follows: in the bar charts, bars are identified by their color and their position, while in the flower glyphs, petals are identified by their color, their position, but also their orientation. The addition of the "orientation" visual variable may increase the associativity and selectivity, facilitating the identification of a given petal, but also the visual selection of similar petal across several glyphs (e.g. the visual selection of the four petals for "respiratory system" ADE in Figure 1). In addition, orientation may support color-blind peoples: the human eye is able to distinguish 12 orientations (as in an analog clock), thus orientation without color is sufficient for identifying the petals in the flower glyph.

We used a light pink color for the "skin and subcutaneous tissue" ADE category. This choice is not culturally independent, but possibly remains the easier for clinicians to remember.

\section{CONCLUSION}

In this paper, we designed four interactive visual analytics for visualizing ADE rates during clinical trials. We compared the associated subjective appreciations. We identified the advantages and disadvantages of each visualization technique. To conclude, two techniques seem interesting: the horizontal stacked bar chart, because it is well-known and it allows displaying short textual labels for labeling the ADE categories, and the flower glyph, because it gives more details on small values. However, we expected that the final user will learn to identify the ADE categories quickly, because there are not numerous (13) and because of the easy-to-remember colors (e.g. yellow for urinary system). As a consequence, the flower glyph seems preferable in the long term. On the contrary, star glyphs seem particularly misleading in our context, and should be avoided.

The main perspective of this work is the integration of the proposed visual analytics in a semantic web platform for drug safety, able to search for clinical trials, to aggregate the results of similar trials, and to display the aggregated ADE rates visually [20]. Another perspective is the use of the proposed area-proportional flower glyph in lieu of the usual lengthproportional flower glyphs, especially when small values are important.

\section{REFERENCES}

[1] D. Keim, G. Andrienko, J. D. Fekete, C. Görg, J. Kohlhammer, and $\mathrm{G}$. Melançon, Information visualization - human-centered issues and perspectives, 2008, no. 4950, ch. Visual Analytics: Definition, Process and Challenges, pp. 154-175.

[2] J. D. Duke, X. Li, and S. J. Grannis, "Data visualization speeds review of potential adverse drug events in patients on multiple medications," $J$ Biomed Inform, vol. 43, no. 2, pp. 326-331, 2009.

[3] J. B. Lamy, H. Berthelot, M. Favre, A. Ugon, C. Duclos, and A. Venot, "Using visual analytics for presenting comparative information on new drugs," J Biomed Inform, vol. 71, pp. 58-69, 2017.

[4] J. B. Lamy, H. Berthelot, C. Capron, and M. Favre, "Rainbow boxes: a new technique for overlapping set visualization and two applications in the biomedical domain," Journal of Visual Language and Computing, vol. 43, pp. 71-82, 2017.

[5] J. B. Lamy, K. Sedki, and R. Tsopra, "Explainable decision support through the learning and visualization of preferences from a formal ontology of antibiotic treatments," J Biomed Inform, vol. 104C, p. 103407, 2020.

[6] R. Tsopra, F. Mechaï, K. Sedki, and J. B. Lamy, "AntibioHelp®, an innovative clinical decision support system for improving antibiotic prescriptions in primary care," pp. 3-5, 2019.

[7] Ward MO, Handbook of data visualization. Berlin Heidelberg: Springer, 2008, ch. Multivariate data glyphs: Principles and practice, pp. 179-198.

[8] R. Borgo, J. Kehrer, D. H. Chung, E. Maguire, R. S. Laramee, H. Hauser, M. Ward, and M. Chen, "Glyph-based Visualization: Foundations, Design Guidelines, Techniques and Applications." in Eurographics, 2013, pp. 39-63.

[9] Lanzenberger M, "The Interactive Stardinates - Design Considerations,' in Proceeding of Human-Computer Interaction (INTERACT'03). Zurich, Switzerland: IOS Press, 2003, pp. 688-693.

[10] J. Fuchs, P. Isenberg, A. Bezerianos, F. Fischer, and E. Bertini, "The Influence of Contour on Similarity Perception of Star Glyphs," IEEE transactions on visualization and computer graphics, vol. 20, no. 12, pp. 2251-2260, 2014.

[11] Chau M, "Visualizing web search results using glyphs: Design and evaluation of a flower metaphor," Acm transactions on management information systems (tmis), vol. 2, no. 1, pp. 1-27, 2011. 


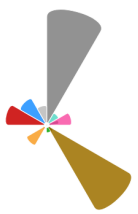

\#1: placebo oral (in 2 daily intakes) for chronic pain, fibromyalgia All: $96.7 \%$ Serious: $3.8 \%$

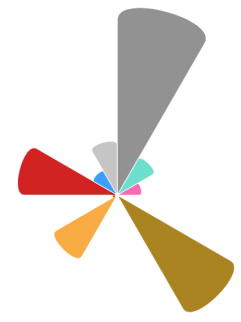
\#2: pregabalin oral (300 mg in 2 daily intakes) for chronic pain, fibromyalgia All: $165.7 \%$ Serious: $1.0 \%$
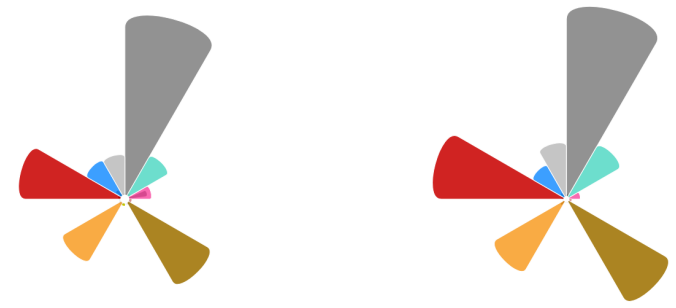
\#4: pregabalin oral (600 mg in 2 daily intakes)
for chronic pain, fibromyalgia
All: $180.1 \%$ Serious: $2.6 \%$

Figure 8. Visualization of the ADE rates of four groups in a clinical trial, using length-proportional flower glyphs.

\begin{tabular}{|c|c|c|c|}
\hline Horizontal stacked bar chart & Vertical stacked bar chart & Flower glyph & Star glyph \\
\hline $\begin{array}{l}+ \text { Well-known diagram } \\
+ \text { Area-proportional } \\
+ \text { Can include textual } \\
\text { labels }\end{array}$ & $\begin{array}{l}+ \text { Well-known diagram } \\
+ \text { Area-proportional }\end{array}$ & $\begin{array}{l}\text { + Area-proportional } \\
\text { + Favor small values } \\
\text { + Visually attractive } \\
\text { + Offer the possibility to } \\
\text { put unclassified ADE in the } \\
\text { center region } \\
\text { + Direction can be used as } \\
\text { hint for organs (e.g. brain } \\
\text { at the top) } \\
\text { - Not well-known }\end{array}$ & $\begin{array}{l}\text { - Not well-known } \\
\text { - Overall shape depends on } \\
\text { axis ordering } \\
\text { - Values surrounded by } \\
\text { zero values are almost } \\
\text { invisible } \\
\text { - Serious ADE are difficult } \\
\text { to read } \\
\text { - Misleading: the surface is } \\
\text { wrongly perceived as } \\
\text { proportional to the ADE } \\
\text { rate }\end{array}$ \\
\hline
\end{tabular}

Positive (+) AND NEGATIVE (-) POINTS OF THE FOUR VISUALIZATION TECHNIQUES.

[12] M. Keck, D. Kammer, T. Gründer, T. Thom, M. Kleinsteuber, A. Maasch, and R. Groh, "Towards glyph-based visualizations for big data clustering," in Proceedings of the 10th international symposium on visual information communication and interaction, 2017, pp. 129-136.

[13] M. Keck, D. Kammer, and R. Groh, "Visual Version Comparison of Multidimensional Data Sets Using Glyphs," in IEEE InfoVis, 2018.

[14] G. Pilato and U. Maniscalco, "A framework based on semantic spaces and glyphs for social sensing on twitter," Procedia computer science, vol. 88, pp. 107-114, 2016.

[15] Y. Anzai and K. Misue, "Evaluation of Effectiveness of Glyphs to Enhance ChronoView," in 2019 23rd international conference information visualisation (iv), 2019, pp. 157-162.

[16] J. Fuchs, D. Jäckle, N. Weiler, and T. Schreck, "Leaf Glyph: Visualizing Multi-dimensional Data with Environmental Cues," in Interna- tional Conference on Information Visualization Theory and Applications (IVAPP), 2015, pp. 195-208.

[17] Ware C, Visual thinking for design. Burlington, USA: Morgan Kaufmann, 2008.

[18] J. Warner, P. Yang, and G. Alterovitz, "Automated synthesis and visualization of a chemotherapy treatment regimen network," Stud Health Technol Inform, vol. 192, pp. 62-6, 2013.

[19] J. Sjöbergh, M. Kuwahara, and Y. Tanaka, "Visualizing clinical trial data using pluggable components," in 16th International Conference on Information Visualisation, 2012, pp. 291-296.

[20] Lamy JB, "A data science approach to drug safety: Semantic and visual mining of adverse drug events from clinical trials of pain treatments," Arxiv preprint arxiv:2006.16910, 2020. 